\title{
The circular loop equation of a cosmic string in Kerr-de Sitter spacetimes
}

\author{
Zhengyan $\mathrm{Gu}^{*} \quad$ Hongbo Cheng ${ }^{\dagger}$ \\ Department of Physics, East China University of Science and Technology, \\ Shanghai 200237, China
}

\begin{abstract}
The equation of cosmic string loops in Kerr-de Sitter spacetimes is derived. Having solved the equation numerically, we find that the loops can evolve except for too small ones in the spacetimes.
\end{abstract}

PACS number(s):

*E-mail address: guzhengyan@citiz.net

${ }^{\dagger}$ E-mail address: hbcheng@public4.sta.net.cn 
Cosmic strings including their formation, evolution and observational effects attracted more attentions in the eighties and much of the nineties $[1,2]$. As linear defects at a symmetry breaking phase transition, cosmic strings can be produced at the end of an inflation. The cosmic strings have several cosmological importance especially two features. One was an adequate explanation for originating the primordial density perturbations which are necessary for galaxies and clusters, and the other is their spacetime metric with deficit angle. However, with expected improvements in observational data, the CMB and WMAP experiments favour the models without cosmic strings due to their weaker tension $G \mu \leq 10^{-6}$, which leads the strings not to seed the large scale structure formation of the universe [3-5].

There has been a recent resurgence of interest in cosmological consequences of cosmic strings for both theoretical and observational reasons. The topological defects including cosmic strings can be inevitably formed at the end of brane inflation [6,7], which provides us with a potential window on the $\mathrm{M}$ theory on the theoretical side [8-10]. On the experimental ones, there are some examples to be found. Sazhin et al discovered a gravitational lens called CSL-1 [11, 12] which is supposed to invoke two images of comparable magnitude of the same giant elliptical galaxy. They found a lot of similar objects in the vicinity. In addition, Schild et al observed and analysed the anomalous brightness fluctuations in a multiple-image lens system such as Q0957+561A, B [13, 14], and the phenomena is interpreted as lensing by an oscillating loops of cosmic string.

It is necessary to continue exploring the evolution of cosmic string loops. Once the cosmic strings formed at any epoch in the history of the universe, curve sections of strings would oscillate under their own force of tension. Although the strings stretch under the influence of the Hubble expansion and the oscillating strings lose energy to gravitational radiation, they collide and intersect to undergo reconnections. The reconnections of long strings and large loops will produce small loops copiously. In general the string networks consist of long strings and closed string loops. The cosmic string loops oscillate with time rather randomly. They are thought as complicated time dependent gravitational source. The gravitationally lensed quasar Q0957+561A, B has been studied intensively for 25 years $[13,14]$. Schild et al put forward an analysis of brightness fluctuations in the system consisting of two quasar images separated by approximately $6^{\prime \prime}$. They are known to be images of the same quasar not only because of the spectroscopic match, but also because the images fluctuate in brightness, and the time delay between fluctuations is always the same. They further suggested that the effect may be due to lensing by an oscillating loop of cosmic string between us and the lensing system because loops of cosmic strings supply quantitative explanations of synchronous variations in the two images of the gravitationally lensed quasar Q0957+561A, B. Therefore the important results such as synchronous variations in the two images with no time delay may be due to the oscillating loops of cosmic string [14]. In a word, the theoretical and observational results support the existence of cosmic string loops. The evolution and fate of cosmic string loops also attracted more attention [1, 2, 15-21]. In some cases like Minkowski space and Robertson-Walker universe the loops will collapse to form black holes or become a long cosmic string instead of keeping 
the oscillating loops. It was showed that the loops of cosmic string in de Sitter spacetimes will keeps up expanding if their initial radii are large enough [19]. That a loop with smaller initial radius may expand in some spacetimes is supported by the observational evidence mentioned above. Clearly it is necessary to investigate the loops in some other spacetimes in order to explore the possibility that the smaller loops of cosmic string can live.

The Kerr-de Sitter spacetimes are currently of great interest. It is a remarkable fact that there are roughly $10^{20}$ rotating black holes in the observable universe, so the surrounding of astrophysically relevant black hole can be described by the Kerr metric [22]. Recently, this kind of spacetime is popular because of its distinct features [23-27]. The Kerr spacetime is stable against massless field perturbations, but it is unstable for massive case because of superradiance. The phenomenon is that the energy of the reflected wave is larger than the incident one in a scattering process. According to the observational evidence that the universe expands faster than we thought [28, 29], it is worth investigating the so called Kerr-de Sitter spacetime describing rotating gravitational sources with a positive cosmological constant. It is necessary to study the Kerr-de Sitter metrics in various directions.

There should be more cosmic string loops existing in our universe in order to explain the astronomical phenomena $[13,14]$. However a large loop will evolve to be a lot of smaller loops and the tiny loops can not inhabit in de Sitter background unless they are very large. The purpose of this paper is to derive the equation of circular loops of cosmic string in the Kerr-de Sitter spacetime. After the loops formation, the circular loops contract under their own tension to form black holes inevitably in the Minkowski, Robertson-Walker universe. In the de Sitter backgrounds, only loops with large initial radii can avoid becoming black holes [19]. It is interesting that for the Kerr-de Sitter cases, the circular loops with initial radius which is much smaller than that in de Sitter spacetime will expand instead of going towards the rotating gravitational source. At first we derive the equations of circular loops of cosmic string in Kerr-de Sitter spacetimes. We solve the equations numerically. The conclusions are emphasized at last.

We start to consider the evolution of cosmic strings in a Kerr-de Sitter spacetime. The metric describing the environment is written as,

$$
d s^{2}=\frac{\Delta_{r}}{\rho^{2}}\left(d t-\frac{a}{\Xi} \sin ^{2} \theta d \phi\right)^{2}-\frac{\rho^{2}}{\Delta_{r}} d r^{2}-\frac{\rho^{2}}{\Delta_{\theta}} d \theta^{2}-\frac{\Delta_{\theta} \sin ^{2} \theta}{\rho^{2}}\left(a d t-\frac{r^{2}+a^{2}}{\Xi} d \phi\right)^{2}
$$

where

$$
\begin{gathered}
\Delta_{r}=\left(r^{2}+a^{2}\right)\left(1-\frac{r^{2}}{L^{2}}\right)-2 m r \\
\Delta_{\theta}=1+\frac{a^{2}}{L^{2}} \cos ^{2} \theta \\
\rho^{2}=r^{2}+a^{2} \cos ^{2} \theta
\end{gathered}
$$




$$
\Xi=1+\frac{a^{2}}{L^{2}}
$$

and $L=\sqrt{\frac{3}{\Lambda}}$ is the de Sitter radius associated with the cosmological constant $\Lambda$. The gravitational source has mass $m$, angular momentum $J=m a$, and an event horizon at $r=r_{+}$, the largest root of $\Delta_{r}$. A free string propagating in a spacetime sweeps out a world sheet which is two-dimensional surface. The Nambu-Goto action is used to describe the motion of string and is given by,

$$
S=-\mu \int d^{2} \sigma\left[\left(\frac{\partial x}{\partial \sigma^{0}} \cdot \frac{\partial x}{\partial \sigma^{1}}\right)^{2}-\left(\frac{\partial x}{\partial \sigma^{0}}\right)^{2}\left(\frac{\partial x}{\partial \sigma^{1}}\right)^{2}\right]^{\frac{1}{2}}
$$

where $\mu$ is the string tension. $\sigma^{a}=(\tau, \sigma)(a=0,1)$ are timelike and spacelike string coordinates respectively. $x^{\mu}(\tau, \sigma)(\mu, \nu=0,1,2,3)$ are the coordinates of the string world sheet in the spacetime.

For simplicity we assume that the string lies in the hypersurface $\theta=\frac{\pi}{2}$, then the spacetime coordinates of the world-sheet parametrized by $\sigma^{0}=t, \sigma^{1}=\varphi$ can be chosen as,

$$
x=\left(c t, r(t, \varphi), \frac{\pi}{2}, \varphi\right)
$$

In the case of planar circular loops, we have $r=r(t)$. According to the metric (1) and coordinates (7), the action (6) is reduced to,

$$
S=-\mu \iint d t d \varphi \sqrt{\Delta}
$$

which leads to the following equation of motion for loops,

$$
\frac{d}{d t} \frac{\partial \Delta}{\partial \dot{r}}-\frac{1}{2 \Delta} \frac{d \Delta}{d t} \frac{\partial \Delta}{\partial \dot{r}}-\frac{\partial \Delta}{\partial r}=0
$$

where

$$
\Delta=\left[\frac{a\left(r^{2}+a^{2}\right)-a \Delta_{r}}{r^{2} \Xi}\right]^{2}-\frac{\left(r^{2}+a^{2}\right)^{2}-\Delta_{r} a^{2}}{\Delta_{r} \Xi^{2}} \dot{r}^{2}+\left(\Delta_{r}-a^{2}\right) \frac{\left(r^{2}+a^{2}\right)^{2}-\Delta_{r} a^{2}}{r^{4} \Xi^{2}}
$$

According to equations (2-5), equation (9) becomes

$$
\begin{aligned}
& 2 \frac{\left(r^{2}+a^{2}\right)^{2}-\Delta_{r} a^{2}}{\Delta_{r} \Xi^{2}}\left\{1+\frac{1}{\Delta} \frac{\left(r^{2}+a^{2}\right)^{2}-\Delta_{r} a^{2}}{\Delta_{r} \Xi^{2}} \dot{r}^{2}\right\} \ddot{r} \\
& +\frac{2\left(r^{2}+a^{2}\right)}{\Delta_{r}^{2} \Xi^{2}}\left[2\left(1+\frac{a^{2}}{L^{2}}\right) r^{3}+2 a^{2}\left(1+\frac{a^{2}}{L^{2}}\right) r-6 m r^{2}+2 m a^{2}\right] \dot{r} \\
& -\frac{\left(r^{2}+a^{2}\right)^{2}-\Delta_{r} a^{2}}{\Delta \Delta_{r} \Xi^{2}}\left\{2 a\left(2 a r \dot{r}-a \frac{d \Delta_{r}}{d t}\right) \frac{\left(r^{2}+a^{2}\right)-\Delta_{r}}{r^{4} \Xi^{2}}\right. \\
& -4\left[a\left(r^{2}+a^{2}\right)-\Delta_{r}\right] \frac{\left(r^{2}+a^{2}\right)-\Delta_{r}}{r^{5} \Xi^{2}} a \dot{r} \\
& -\left(\frac{1}{\Delta_{r} \Xi^{2}}\left[4\left(r^{2}+a^{2}\right) r \dot{r}-\frac{d \Delta_{r}}{d t} a^{2}\right]-\frac{\left(r^{2}+a^{2}\right)^{2}-\Delta_{r} a^{2}}{\Delta_{r}^{2} \Xi^{2}} \frac{d \Delta_{r}}{d t}\right) \dot{r}^{2} \\
& +\frac{d \Delta_{r}}{d t} \frac{\left(r^{2}+a^{2}\right)^{2}-\Delta_{r} a^{2}}{r^{4} \Xi^{2}}+\frac{\Delta_{r}-a^{2}}{r^{4} \Xi^{2}}\left[4\left(r^{2}+a^{2}\right) r \dot{r}-\frac{d \Delta_{r}}{d t} a^{2}\right]
\end{aligned}
$$




$$
\begin{aligned}
&\left.-4\left(\Delta_{r}-a^{2}\right) \frac{\left(r^{2}+a^{2}\right)^{2}-\Delta_{r} a^{2}}{r^{5} \Xi^{2}} \dot{r}\right\} \\
&+\frac{4 a^{2}}{r^{3} \Xi^{2}}\left(\frac{r^{3}}{L^{2}}+\frac{a^{2}}{L^{2}} r+2 m\right) \\
&-\frac{r^{2}+a^{2}}{\Delta_{r}^{2} \Xi^{2}}\left[2\left(1+\frac{a^{2}}{L^{2}}\right) r^{3}+2 a^{2}\left(1+\frac{a^{2}}{L^{2}}\right) r-6 m r^{2}+2 m a^{2}\right] \dot{r}^{2} \\
&+\frac{1}{r^{3} \Xi^{2}}\left[-\frac{4 r^{3}}{L^{2}}+2\left(1-\frac{a^{2}}{L^{2}}\right) r-2 m\right]\left[\left(1+\frac{a^{2}}{L^{2}}\right) r^{3}+\left(1+\frac{a^{2}}{L^{2}}\right) a^{2} r+2 m a^{2}\right] \\
& \quad+\frac{1}{r^{3} \Xi^{2}}\left[\frac{r^{3}}{L^{2}}-\left(1-\frac{a^{2}}{L^{2}}\right) r+2 m\right]\left[2\left(1+\frac{a^{2}}{L^{2}}\right) a^{2} r+6 m a^{2}\right]=0
\end{aligned}
$$

where

$$
\frac{d \Delta_{r}}{d t}=\left[-\frac{4 r^{3}}{L^{2}}+2\left(1-\frac{a^{2}}{L^{2}}\right) r-2 m\right] \dot{r}
$$

here the functions $\Delta_{r}, \Xi$ are denoted in (2) and (5) respectively.

We shall consider the solution with $\dot{r}(0)=0$, and we assume that loops are static when $t=0$, the time of formation of loops, by neglecting the peculiar velocities of loops. For the circular loops in de Sitter spacetimes, A. L. Larsen showed that the loops with larger initial radius like $r(0)>0.707 L$ expand instead of becoming the black hole [19]. If the initial radius $r(0)<0.707 L$, the circular loop will collapse to form black hole in that spacetime. Here $L$ is the de Sitter size. It was also pointed out that the cosmic string loops aligned perpendicular to the spin axis of a Kerr source will contract to be captured by the gravitational source at last. We solve the equation of motion (11) numerically by a Runge-Kutta algorithm to research on the evolution of cosmic string loops in the Kerr-de Sitter spacetimes. The evolution curves $\frac{r(t)}{L}=R(t)$ are plotted in figure 1 versus the different $\frac{r(0)}{L}=R(0), 0<R(0)<1$. The numerical results show that there exist special parameter $R_{0}$ for a fixed $m$ and a fixed relative angular momentum denoted as $A=\frac{a}{L}$. If $R(0)>R_{0}$, the loop will expand or they will approach to the gravitational source instead of surviving. The influence from source mass $m$ has been studied [19]. The relation between the special parameter $R_{0}$ and $A=\frac{a}{L}$ for a fixed parameter like $M=\frac{m}{L}$ is shown in figure 2. The special parameter $R_{0}$ is decreasing when the relative angular momentum $A$ is increasing. Therefore, the influence of rotating gravitational source, represented by parameter $A$, gives rise to decreasing of initial radius of cosmic string loops. Thus, around the rotating gravitational source in the presence of positive cosmological constant, the smaller cosmic string loops can become large to evolove instead of contracting to approach to the source. It is interesting for us to find that the required initial radii which lead the loops exist not to move towards the source in Kerr-de Sitter backgrounds can be smaller than those in de Sitter spacetimes.

According to the observational results, there should exist more cosmic string loops in our Universe $[13,14]$. Here we report the evolution of planar circular loops of cosmic string in the surrounding of a rotating body with positive cosmological constant to investigate the fate of loops. The loops with relatively smaller radius at the beginning of formation expand and will not collapse in the environment. With colliding and intersecting of long strings and large loops, a lot of tiny 
loops are generated continuously. In the de Sitter world most of the loops become black holes [19], which means that few loops can survive. Our findings on the evolution and existence of small loops indicate that there may be a considerable number of loops of cosmic string in our Universe due to a numerous number of rotating black holes and positive cosmological constant. The same process that long strings and large loops change into more small loops and most of them expand and intersect into small loops go through again and again, which can keep us observing the effect of cosmic string loops.

The main result of this paper is equation(11), the circular loop equation for a cosmic string evolving in the hypersurface with $\theta=\frac{\pi}{2}$ in the Kerr-de Sitter spacetime. A remarkable solution to this equation is that a loop may never contract towards the source if its initial size is larger than a special parameter denoted as $R_{0}$. Figure 2 shows that the special parameter depends on the angular momentum of the rotating gravitational source, larger the angular momentum, smaller the special parameter. According to figure 2, all special parameters for cosmic string loops around rotating gravitational sources with positive cosmological constant are smaller than $0.707 L$, the special parameter for the loops in the de Sitter spacetime obtained in [19]. Therefore a lot of cosmic string loops including some smaller ones can evolve to survive in the Kerr-de Sitter spacetime. The general evolution of cosmic string loops requires further research.

\section{Acknowledge}

This work is supported by the Basic Theory Research Fund of East China University of Science and Technology, grant No. YK0127312 and partly supported by the Shanghai Municipal Science and Technology Commission No.04dz05905.. 


\section{References}

[1] A. Vielenkin, E. P. S. Shellard, Cosmic Strings and Other Topological Defects, Cambridge University Press 1994.

[2] M. B. Hindmarsh, T. W. B. Kibble, Rep. Prog. Phys. 58, 477(1995)

[3] L. Pogosian, M. C. Wyman, I. Wasserman, "Observational constraints on cosmic strings: Bayesian analysis in a three dimensional parameter space", astro-ph/0403268.

[4] E. Jeong, G. F. Smoot, "Search for cosmic strings in CMB anisotropies", astro-ph/0406432.

[5] M. V. Sazhin et. al., "Lense candidates in the Capodimonte Deep Field in the vincinity of the CSL1 object", astro-ph/0406516.

[6] S. Sarangi, S. H. H. Tye, Phys. Lett. B536, 185(2002).

[7] L. Pogosian, S. H. H. Tye, I. Wasserman, M. Wyman, Phys. Rev. D68, 023506(2003).

[8] N. T. Jones, H. Stoica, S. H. H. Tye, Phys. Lett. B563, 6(2003).

[9] G. Dvali, A. Vilenkin, JCAP 0403, 010(2004).

[10] M. G. Jackson, N. T. Jones, J. Polchinski, hep-th/0405229.

[11] M. Sazhin, G. Longo, M. Capaccioli, J. M. Alcala, R. Silvotti, G. Covone, O. Khovanskaya, M. Pavlov, M. Pannella, M. Radovich, V. Testa, MNRAS 343, 353(2003).

[12] M. Sazhin, O. Khovanskaya, M. Capaccioli, G. Longo, J. M. Alcala, R. Silvotti, M. V. Pavlov, astro-ph/0406434.

[13] A. de Laix, T. Vachaspati, Phys. Rev. D54, 4780(1996).

[14] R. Schild, I. S. Masnyak, B. I. Hnatyk, V. I. Zhdanov, "Anomalous fluctuations in observations of Q0957+561A. B: Smoking gun of a cosmic string?", astro-ph/0406434.

[15] T. W. B. Kibble, Phys. Rep. 67, 183(1980).

[16] A. Vilenkin, Phys. Rep. 121, 263(1985).

[17] X. Li, J. Zhang, Phys. Lett. B312, 62(1993).

[18] J. Garriga, A. Vilenkin, Phys. Rev. D47, 3265(1993).

[19] A. L. Larsen, Phys. Rev. D50, 2623(1994)

M. R. Anderson, The mathematical theory of cosmic strings - cosmic strings in the wire approximation, IOP Publishing Ltd. 2003.

[20] X. Li, Hongbo Cheng, Class. Quantum Grav. 13, 225(1996). 
[21] M. P. Dabrowski, A. L. Larsen, Phys. Rev. D57, 5108(1998).

[22] R. P. Kerr, Phys. Rev. Lett. 11, 237(1963).

[23] H. Furuhashi, Y. Nambu, gr-qc/0402037.

[24] Z. Stuchlik, P. Slany, Phys. Rev. D69, 064001(2004).

[25] M. Vasudevan, K. A. Stevens, D. N. Page, gr-qc/0405125.

[26] G. W. Gibbons, H. Lu, D. N. Page, C. N. Pope, hep-th/0409155.

[27] G. W. Gibbons, M. J. Perry, C. N. Pope, hep-th/0408217.

[28] S. Perlmutter, et. al., Astrophys. J. 517, 565(1999).

[29] S. Perlmutter, Int. J. Mod. Phys. A15S1, 715(2000). 


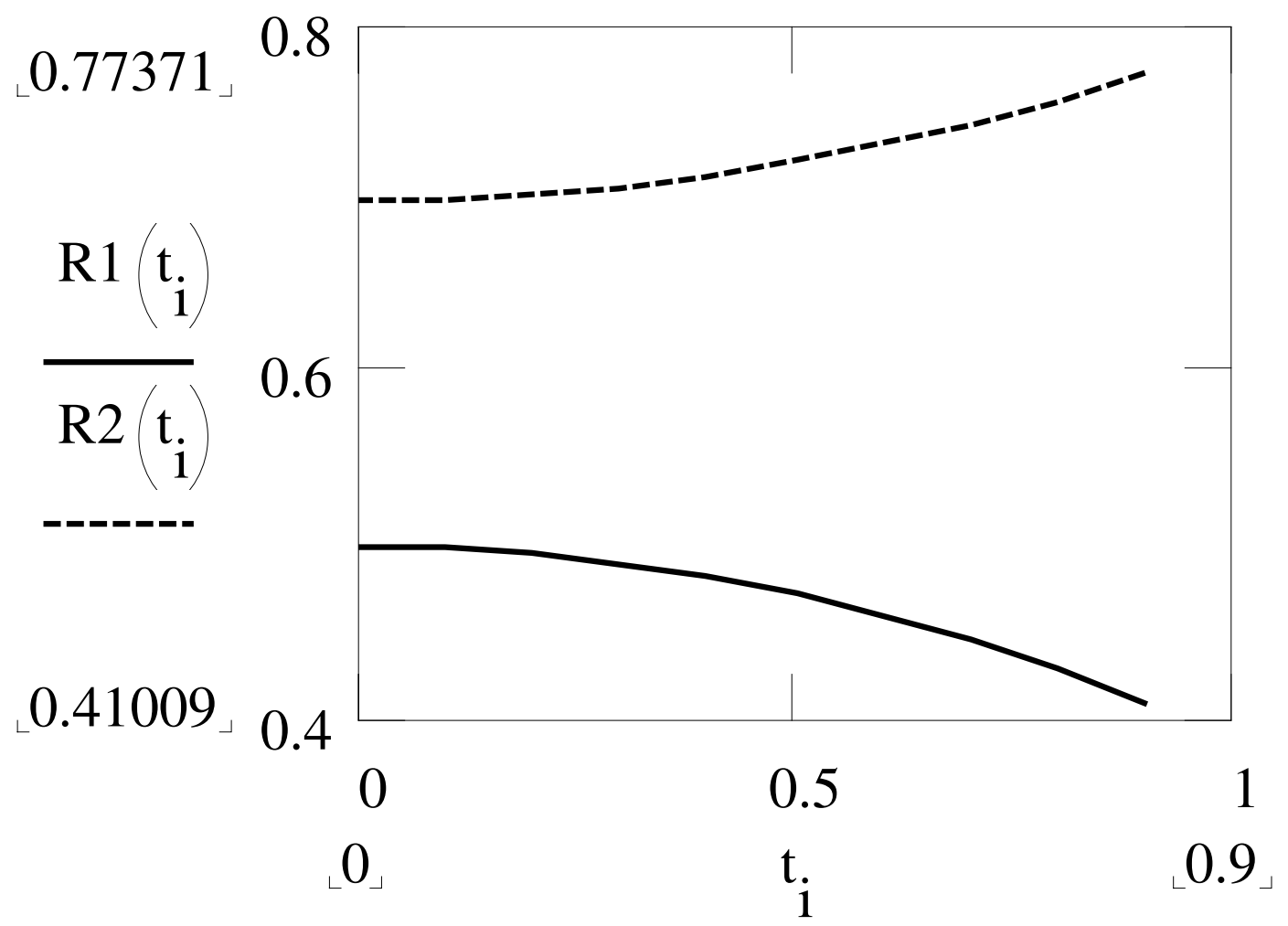

Figure 1: The evolution of loop size versus the different initial radii, solid line for $R(0)=0.5$ and dashed line for $R(0)=0.7$ 


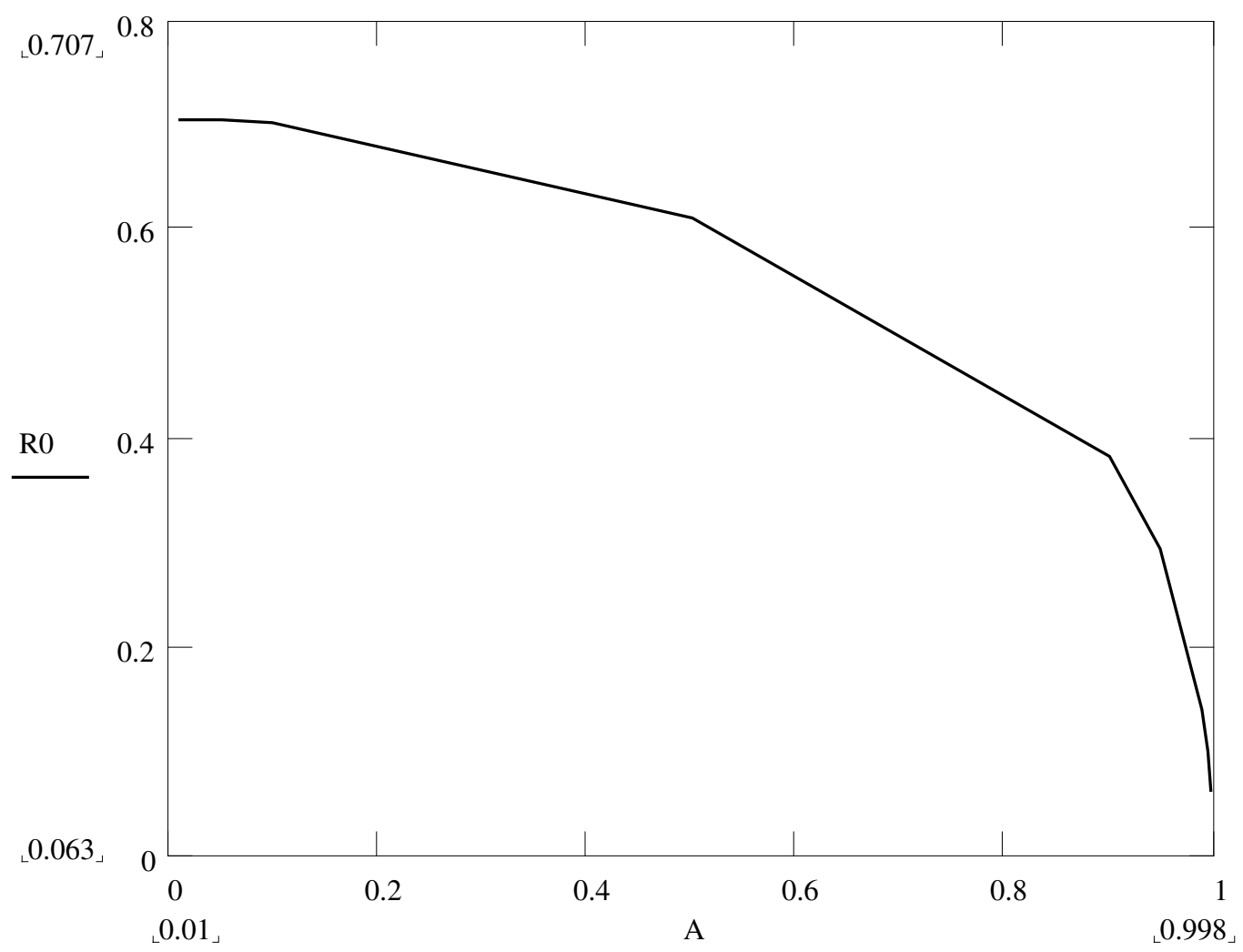

Figure 2: The special parameter $R_{0}$ as a function of the relative angular momentum $A$ for $\frac{m}{L}=0.0001$ 